\title{
Peritoneal Infection, CTCAE
}

National Cancer Institute

\section{Source}

National Cancer Institute. Peritoneal Infection, CT CAE. NCI Thesaurus. Code C143753.

A disorder characterized by an infectious process involving the peritoneum. 